\title{
Avaliação físico-química e estabilidade do ácido ascórbico em sucos de frutas comercializados no município de Frutal, Minas Gerais
}

\section{Evaluation physical-chemical and stability of ascorbic acid in fruit juices marketed in city of Frutal, Minas Gerais, Brazil}

\author{
Luiza Farias da Silva Souza ${ }^{1}$, Laura Ferreira Domingos ${ }^{2}$, Vera Lúcia da Silva Farias ${ }^{3}$, Débora Maria Moreno Luzia ${ }^{4 *}$
}

Resumo: Objetivou-se analisar a avaliação físico-química e a estabilidade do ácido ascórbico presentes em diferentes marcas de sucos de frutas pasteurizados e envasados em embalagem Tetra Pak comercializados no município de Frutal no Estado de Minas Gerais. Avaliou-se as características físico-químicas ( $\mathrm{pH}$, acidez titulável e sólidos solúveis totais em ${ }^{\circ}$ Brix) e a estabilidade do ácido ascórbico pelo método de Tillmans em sucos de frutas, durante um período de 21 dias de armazenamento sob refrigeração. Para isso, os sucos foram adquiridos entre os meses de maio e junho de 2015, no comércio local e em seguida, conservados em suas embalagens até o momento das análises. De acordo com os resultados obtidos com a avaliação físico-química, o pH, a acidez titulável e os sólidos solúveis totais, apresentaram variações durante o armazenamento. Porém, a maioria das amostras analisadas de sucos atendeu aos parâmetros mínimos de qualidade exigidos pelo Ministério da Agricultura. Quanto à estabilidade do ácido ascórbico, verificou-se uma maior estabilidade para os sucos de laranja (marca A e C) e de maracujá (marca B). Assim, pode-se concluir que houve retenção do ácido ascórbico nos sucos de frutas após 21 dias de armazenamento sob refrigeração, com possível minimização das perdas de vitamina C durante o processamento, indicando-r boa qualidade dos sucos comercializados no município de Frutal, Minas Gerais.

Palavras-chave: Vitamina C; Frutas; Legislação brasileira.

Abstract: The objective was to analyzethe physical-chemical evaluation and the stability of ascorbic acid present in different brands of unpasteurized fruit juices and bottled in Tetra Pak packaging marketed in city of Frutal- Minas Gerais State. The objectives were to evaluate physical-chemical characteristics ( $\mathrm{pH}$, titratable acidity and total soluble solids in $\left.{ }^{\circ} \mathrm{Brix}\right)$ and the stability of ascorbic acid by Tillmans method in fruit juices for a period of 21 days of storage under refrigeration. For this, the juices were purchased between May and June 2015, in the local market and then preserved in their containers until the time of analysis. According to the results obtained for the physical-chemical, pH, titration acidity and total soluble solids, showed variations during storage. However, most of the samples of juices meets the minimum quality parameters required by Ministry of Agriculture. The stability of ascorbic acid is found greater stability for orange juice (brand A and C) and passion fruit (brand B). Thus, it can be concluded that there was retention of ascorbic acid in fruit juices after 21 days of refrigerated storage, with possible minimization of losses of vitamin C during processing, indicating good quality of juices marketed in city of Frutal, Minas Gerais.

Key words: Vitamin C; Fruits; Brazilian legislation.

\footnotetext{
*Autor para correspondência

Recebido para publicação em 06/04/2016; aprovado em 04/09/2017

${ }^{1}$ Estudante do Ensino Fundamental da Escola Estadual de Frutal, Minas Gerais. E-mail: lu.lu.farias@ hotmail.com

${ }^{2}$ Tecnóloga em Alimentos, Graduada pela Universidade do Estado de Minas Gerais. E-mail: laurafdomingos@gmail.com

${ }^{3}$ Bióloga, Professora da Universidade do Estado de Minas Gerais. E-mail: verlucbio@ yahoo.com.br

${ }^{4 *}$ Nutricionista, Professora da Universidade do Estado de Minas Gerais. E-mail: deboramaria_moreno@yahoo.com.br
} 


\section{INTRODUÇÃO}

As frutas possuem um alto valor nutritivo, sendo seu consumo diário indicado por serem fontes de vitaminas, minerais e carboidratos. As mesmas podem ser consumidas in natura ou utilizadas em várias formas de preparações.

$\mathrm{O}$ mercado brasileiro de suco de fruta industrializado vem crescendo rapidamente nos últimos anos, sendo o suco de fruta pronto para beber o principal responsável por essa expansão, que vem acompanhando a tendência mundial de consumo de bebidas que oferecem saúde, conveniência, sabor, inovação e prazer (ROSA et al., 2006).

Os sucos devem atender à legislação específica, estando de acordo com definição, classificação, registro, padronização e requisitos de qualidade, devendo também atender à legislação sobre rotulagem de alimentos embalados (BRASIL, 1994; BRASIL, 1997). A legislação brasileira na área de alimentos é regida pelo Ministério da Saúde, por intermédio da Agência Nacional de Vigilância Sanitária (ANVISA) e pelo Ministério da Agricultura, Pecuária e Abastecimento (MAPA). E ainda, de acordo com a legislação brasileira, suco ou sumo é definido como: "bebida não fermentada, não concentrada e não diluída, destinada ao consumo, obtida da fruta sã e madura, ou parte do vegetal de origem, por processo tecnológico adequado, submetida a tratamento que assegure a sua apresentação e conservação até o consumo." (BRASIL, 1997).

Os sucos são consumidos e apreciados em todo mundo, não só pelo seu sabor, mas também por serem fontes naturais de nutrientes. Segundo Castro et al. (2007), os sucos de frutas são fontes de carboidratos, , minerais e vitaminas, principalmente, ácido ascórbico (vitamina C). Essa vitamina hidrossolúvel participa da síntese de colágeno, atua como antioxidante, facilita a absorção de ferro e zinco no trato intestinal, promove resistência a infecções, além de ajudar nos processos de cicatrização (MAHANESCOTT-STUMP, 2005). Porém, o teor vitamínico contido no suco é diferente para cada variedade de fruta.

Em alimentos, o ácido ascórbico é usado como índice de qualidade nutricional dos produtos derivados de frutas e vegetais porque, comparado a outros nutrientes, esta vitamina é mais sensível à degradação por exposição ao calor, além de sofrer alterações aceleradas pela presença de oxigênio e pelo $\mathrm{pH}$ do meio, entre outras condições. Assim, o ácido ascórbico está sujeito a perdas significativas ao longo do armazenamento ou do processamento (ÖZKAN et al., 2004; SPINOLA et al., 2013).

A metodologia padrão 967.26 da AOAC (Association of Official Analytical Chemists), para determinação de ácido ascórbico em bebidas, consiste no método titulométrico envolvendo o 2,6-diclorofenol-indofenol (DCFI), conhecido como Reagente de Tillmans (HOROWITZ, 2010).

A literatura apresenta vários relatos sobre a estabilidade do ácido ascórbico em sucos de frutas. Segundo Lima (2007), em estudo sobre a degradação de vitamina $\mathrm{C}$ em suco de caju industrializado e cajuína, analisadas no dia da abertura da embalagem e após 3, 7 e 11 dias da abertura, houve uma relação linear $(r=0,985)$ entre os teores de vitamina $C$ após 7 e 11 dias de abertura da embalagem, indicando a persistência do padrão de oxidação de vitamina $C$. Apenas a cajuína de laboratório manteve-se com teor de vitamina $\mathrm{C}$ acima do limite mínimo com 7 dias de abertura da embalagem.
Em estudo realizado por Danieli et al. (2009) sobre a determinação de vitamina $\mathrm{C}$ em amostras de sucos in natura e amostras comerciais de suco de laranja pasteurizado e envasado em embalagem Tetra Pak, concluíram que a quantidade de ácido ascórbico em ambas as amostras foi superior ao limite mínimo estabelecido pelo Ministério da Agricultura e do Abastecimento.

Tendo em vista o alto consumo de sucos de frutas tropicais e o interesse em reduzir as perdas nutricionais durante o seu armazenamento, o objetivo deste foi analisar a avaliação físico-química e a estabilidade do ácido ascórbico presentes em diferentes marcas de sucos de frutas pasteurizados e envasados em embalagem Tetra Pak comercializados no município de Frutal (MG)

\section{MATERIAL E MÉTODOS}

As amostras de sucos de frutas pasteurizados e envasados em embalagem Tetra Pak das seguintes frutas: caju, laranja, maracujá e uva, de três diferentes empresas produtoras, foram adquiridas em diferentes estabelecimentos do comércio local da cidade de Frutal (MG), entre os meses de maio e junho de 2015 e transportadas para posterior análises no Laboratório de Análises Físico-Química da Universidade do Estado de Minas Gerais (UEMG)

As determinações analíticas foram realizadas em intervalos de tempo distribuídos da seguinte forma: no dia da abertura da embalagem (tempo 0) e após 7, 14 e 21 dias de armazenamento sob refrigeração a $7 \pm 2^{\circ} \mathrm{C}$.

Para a determinação de $\mathrm{pH}$, utilizou-se peagâmetro, com uma precisão de 0,1 , previamente calibrado de acordo com as recomendações do fabricante, por meio de tampões com $\mathrm{pH}=7 \mathrm{e} \mathrm{pH}=4$. A quantidade de $20 \mathrm{~mL}$ de cada produto foi transferida para um Becker, seguindo-se da imersão do eletrodo e subsequente leitura e registro dos valores obtidos. Após cada aferição, o eletrodo era lavado com água destilada e seco com papel toalha, com o intuito de não levar resquícios de uma amostra para a outra. Entre cada avaliação foi dado um intervalo de 10 minutos;

Para acidez titulável (\% ácido de acordo com o suco), pipetou-se $10,00 \mathrm{~mL}$ de cada amostra que foi transferida para erlenmeyers de $125 \mathrm{~mL}$ com o auxílio de $50 \mathrm{~mL}$ de água. Em seguida, adicionou-se 3 gotas da solução de fenolftaleína e titulou-se as amostras com solução de $\mathrm{NaOH} 0,1 \mathrm{~N}$ (padronizado com Biftalato de Potássio). A titulação foi realizada até coloração rósea, segundo metodologia do Instituto Adolfo Lutz (2008). Os teores de acidez total titulável foram quantificados de acordo com o ácido presente na fruta, neste caso a acidez titulável em função do ácido cítrico foi determinada para os sucos de caju, laranja e maracujá e, o ácido tartárico para o suco uva.

Para sólidos solúveis totais $\left({ }^{\circ} \mathrm{Brix}\right)$, utilizou-se as medidas do grau Brix obtidas por meio do refratômetro de Abbé (BioBrix, modelo 2WAJ - 0 a 95\% Brix), previamente calibrado à temperatura ambiente com água deionizada (índice de refração $=1,3330$ e $0^{\circ}$ Brix a $20^{\circ} \mathrm{C}$ ) e, em seguida, foi procedida à leitura das amostras. Entre as avaliações deuse um intervalo de 10 minutos (AOAC, 2005).

A determinação de ácido ascórbico $(\mathrm{mg} / 100 \mathrm{MI})$, foi realizado pelo método titulométrico de Tillmans (HOROWITZ, 2010), $25 \mathrm{~mL}$ de cada amostra foi titulada com solução de 2,6-diclorofenol-indofenol, previamente padronizada com solução de ácido ascórbico. 
Para os resultados obtidos das determinações analíticas, , foram considerados os seguintes fatores: amostras de sucos de frutas (caju, laranja, maracujá e uva) e tempos de armazenamento $(0,7,14$ e 21 dias). Os resultados obtidos foram submetidos às análises de variância (ANOVA) para determinar a influência do tempo de armazenamento sobre a alteração dos sucos de frutas. $\mathrm{O}$ experimento foi realizado em esquema fatorial $4 \times 4$, no delineamento inteiramente casualizado. A análise de variância e as diferenças entre as médias foram testadas a $5 \%$ de probabilidade pelo teste de Tukey, através do programa ASSISTAT, versão 7.7 beta. (SILVA; AZEVEDO, 2002).

\section{RESULTADOS E DISCUSSÃO}

A medida do $\mathrm{pH}$ é importante na análise de alimentos industrializados, a base de frutas, uma vez que está relacionada a retenção do sabor-odor de produtos de frutas, a estabilidade de corantes artificiais bem como a verificação do estado de maturação das frutas (CECCHI, 2003). Na Tabela 1 observa-se os valores de $\mathrm{pH}$ das amostras de sucos de frutas, durante os 21 dias de armazenamento, sob refrigeração a $7 \pm$ $2{ }^{\circ} \mathrm{C}$.
Os resultados das análises de $\mathrm{pH}$, obtidos logo após a abertura das embalagens (tempo 0 dia) variou de 2,76 a 4,04, e no final do tempo de armazenamento durante 21 dias, foi de 2,74 a 3,76 diferindo pelo teste de Tukey a 5\% de probabilidade $(\mathrm{p}<0,05)$, respectivamente. Segundo CorrêaNeto e Faria (1999) sucos de frutas cítricas apresentam pH com variação de 3,40 a 4,00.

Durante o período de armazenamento das amostras de sucos ocorreram oscilações no $\mathrm{pH}$. Segundo Silva et al. (2005) isso pode ocorrer devido a uma possível decomposição por hidrólise, oxidação ou fermentação que podem modificar a concentração de íons hidrogênio e, consequentemente, a acidez de produtos elaborados a base de frutas.

A legislação brasileira (Instrução Normativa $n^{\circ} 01$, de 7 de janeiro de 2000) para sucos de frutas tropicais não estabelece um valor mínimo de $\mathrm{pH}$ como padrão de identidade e qualidade (BRASIL, 2000).

A acidez total titulável é um importante parâmetro para indicar a adequação dos processos de conservação de bebidas à base de frutas. A decomposição por hidrólise, a oxidação ou a fermentação podem modificar a concentração de íons hidrogênio e, consequentemente, a acidez dos produtos elaborados a base de frutas (INSTITUTO ADOLFO LUTZ, 2008).

Tabela 1. Valores médios de pH em sucos de frutas de diferentes marcas durante o armazenamento sob refrigeração.

\begin{tabular}{lcccc}
\hline \multirow{2}{*}{ Amostras } & \multicolumn{4}{c}{ Tempo de armazenamento sob refrigeração (dias) } \\
\cline { 2 - 5 } Marca A & 0 & 7 & 14 & 21 \\
Caju & $3,64 \pm 0,06^{\mathrm{aA}}$ & $3,60 \pm 0,10^{\mathrm{aB}}$ & $3,59 \pm 0,21^{\mathrm{bB}}$ & $3,59 \pm 0,10^{\mathrm{aB}}$ \\
Laranja & $3,54 \pm 0,10^{\mathrm{bC}}$ & $3,53 \pm 0,12^{\mathrm{bC}}$ & $3,61 \pm 0,18^{\mathrm{aA}}$ & $3,45 \pm 0,12^{\mathrm{bB}}$ \\
Maracujá & $2,76 \pm 0,12^{\mathrm{dA}}$ & $2,75 \pm 0,15^{\mathrm{dA}}$ & $2,74 \pm 0,17^{\mathrm{dA}}$ & $2,74 \pm 0,24^{\mathrm{dA}}$ \\
Uva & $3,04+0,22^{\mathrm{cA}}$ & $3,06 \pm 0,12^{\mathrm{cA}}$ & $3,04 \pm 0,14^{\mathrm{cA}}$ & $2,93 \pm 0,17^{\mathrm{cB}}$ \\
Marca B & & & & \\
Caju & $3,59 \pm 0,11^{\mathrm{aA}}$ & $3,60 \pm 0,15^{\mathrm{aA}}$ & $3,59 \pm 0,11^{\mathrm{aA}}$ & $3,38 \pm 0,10^{\mathrm{aB}}$ \\
Laranja & $3,39 \pm 0,12^{\mathrm{bA}}$ & $3,31 \pm 0,22^{\mathrm{bB}}$ & $3,34 \pm 0,16^{\mathrm{bB}}$ & $3,27 \pm 0,08^{\mathrm{bC}}$ \\
Maracujá & $3,16 \pm 0,10^{\mathrm{cA}}$ & $3,13 \pm 0,16^{\mathrm{cA}}$ & $3,11 \pm 0,15^{\mathrm{cA}}$ & $3,06 \pm 0,20^{\mathrm{cB}}$ \\
Uva & $2,90+0,08^{\mathrm{dA}}$ & $2,87 \pm 0,10^{\mathrm{dA}}$ & $2,90 \pm 0,14^{\mathrm{dA}}$ & $2,77 \pm 0,16^{\mathrm{dB}}$ \\
Marca C & & & & \\
Caju & $4,04 \pm 0,11^{\mathrm{aA}}$ & $3,89 \pm 0,15^{\mathrm{aB}}$ & $3,88 \pm 0,11^{\mathrm{aB}}$ & $3,76 \pm 0,10^{\mathrm{aC}}$ \\
Laranja & $3,55 \pm 0,12^{\mathrm{bA}}$ & $3,52 \pm 0,22^{\mathrm{bAB}}$ & $3,50 \pm 0,16^{\mathrm{bAB}}$ & $3,48 \pm 0,08^{\mathrm{bB}}$ \\
Maracujá & $3,57 \pm 0,10^{\mathrm{bA}}$ & $3,50 \pm 0,16^{\mathrm{bB}}$ & $3,45 \pm 0,15^{\mathrm{cC}}$ & $3,38 \pm 0,20^{\mathrm{cD}}$ \\
Uva & $3,41+0,08^{\mathrm{cA}}$ & $3,24 \pm 0,10^{\mathrm{cB}}$ & $3,20 \pm 0,14^{\mathrm{dC}}$ & $3,16 \pm 0,16^{\mathrm{dD}}$ \\
\hline
\end{tabular}

*Médias \pm desvios padrões das análises realizadas em triplicata.

a, b... (coluna): médias seguidas de mesma letra minúscula não diferem pelo teste de Tukey $(\mathrm{p}>0,05)$.

A, B... (linha): médias seguidas de mesma letra maiúscula não diferem pelo teste de Tukey $(\mathrm{p}>0,05)$.

Na Tabela 2 verifica-se os valores médios para a acidez total titulável nas amostras de sucos de frutas, durante os 21 dias de armazenamento sob refrigeração a $7 \pm 2{ }^{\circ} \mathrm{C}$. De acordo com os resultados observa-se que para a maioria dos sucos a acidez diminuiu ao longo do período de armazenamento, com diferença significativa pelo teste de Tukey $(\mathrm{p}<0,05)$. O maior índice de acidez, independente da marca pode ser verificado para os sucos de maracujá, laranja e uva desde o tempo inicial até o final. Já o suco de caju para as marcas A, $\mathrm{B}$ e $\mathrm{C}$, foi o que apresentou os menores índices com 0,65 ; 0,35 e 0,28 de ácido cítrico no tempo inicial e 0,38;0,32 e 0,24\% de ácido cítrico após 21 dias, sob armazenamento a $7^{\circ} \mathrm{C}$, respectivamente. A legislação brasileira vigente estabelece um valor mínimo para este parâmetro de $0,30 \%$ para suco de caju; $7,0 \%$ para suco de laranja; $2,50 \%$ para suco de maracujá e 0,41\% para suco de uva (BRASIL, 2000).

Os valores dos sólidos solúveis totais (SST) medidos por refratometria e expressos em ${ }^{\circ}$ Brix são utilizados como índice de açúcar totais nos frutos e seu grau de maturidade.

Esse índice representa um importante atributo de qualidade, contribuindo, dessa forma, para o aroma característico dos sucos. Na agroindústria esse parâmetro é usado para intensificar o controle de matéria-prima, processo e qualidade do produto final.

Observa-se na Tabela 3 os valores médios obtidos para os sólidos solúveis totais nas amostras de sucos de frutas, durante os 21 dias de armazenamento sob refrigeração a $7 \pm$ $2{ }^{\circ} \mathrm{C}$. 
Tabela 2. Valores médios de acidez total titulável $(\%)$ em sucos de frutas de diferentes marcas durante o armazenamento sob refrigeração.

\begin{tabular}{|c|c|c|c|c|}
\hline \multirow{2}{*}{ Amostras } & \multicolumn{4}{|c|}{ Tempo de armazenamento sob refrigeração (dias) } \\
\hline & 0 & 7 & 14 & 21 \\
\hline \multicolumn{5}{|l|}{ Marca A } \\
\hline Caju & $0,46 \pm 0,16^{\mathrm{cA}}$ & $0,42 \pm 0,06^{\mathrm{dB}}$ & $0,41 \pm 0,20^{\mathrm{dB}}$ & $0,38 \pm 0,10^{\mathrm{cC}}$ \\
\hline Laranja & $0,82 \pm 0,12^{\mathrm{bA}}$ & $0,82 \pm 0,10^{\mathrm{bA}}$ & $0,82 \pm 0,14^{\mathrm{bA}}$ & $0,81 \pm 0,18^{\mathrm{aA}}$ \\
\hline Maracujá & $0,90 \pm 0,10^{\mathrm{aA}}$ & $0,90 \pm 0,15^{\mathrm{aA}}$ & $0,88 \pm 0,10^{\mathrm{aB}}$ & $0,81 \pm 0,20^{\mathrm{aC}}$ \\
\hline Uva & $0,83+0,20^{\mathrm{bA}}$ & $0,54 \pm 0,10^{\mathrm{cB}}$ & $0,54 \pm 0,12^{\mathrm{cB}}$ & $0,54 \pm 0,10^{\mathrm{bB}}$ \\
\hline \multicolumn{5}{|l|}{ Marca B } \\
\hline Caju & $0,35 \pm 0,08^{\mathrm{cA}}$ & $0,35 \pm 0,16^{\mathrm{cA}}$ & $0,33 \pm 0,20^{\mathrm{cB}}$ & $0,32 \pm 0,08^{\mathrm{dB}}$ \\
\hline Laranja & $0,88 \pm 0,14^{\mathrm{aA}}$ & $0,87 \pm 0,12^{\mathrm{aA}}$ & $0,82 \pm 0,17^{\mathrm{aB}}$ & $0,78 \pm 0,12^{\mathrm{aC}}$ \\
\hline Maracujá & $0,77 \pm 0,12^{\mathrm{bA}}$ & $0,76 \pm 0,14^{\mathrm{bA}}$ & $0,70 \pm 0,15^{\mathrm{bB}}$ & $0,69 \pm 0,20^{\mathrm{cB}}$ \\
\hline Uva & $0,85+0,10^{\mathrm{aA}}$ & $0,76 \pm 0,12^{\mathrm{bB}}$ & $0,72 \pm 0,10^{\mathrm{bC}}$ & $0,70 \pm 0,14^{\mathrm{bC}}$ \\
\hline \multicolumn{5}{|l|}{ Marca C } \\
\hline Caju & $0,28 \pm 0,12^{\mathrm{cA}}$ & $0,27 \pm 0,18^{\mathrm{cA}}$ & $0,26 \pm 0,16^{\mathrm{dA}}$ & $0,24 \pm 0,10^{\mathrm{dA}}$ \\
\hline Laranja & $0,96 \pm 0,10^{\mathrm{aA}}$ & $0,94 \pm 0,10^{\mathrm{aA}}$ & $0,93 \pm 0,14^{\mathrm{aA}}$ & $0,87 \pm 0,12^{\mathrm{aB}}$ \\
\hline Maracujá & $0,97 \pm 0,12^{\mathrm{aA}}$ & $0,94 \pm 0,12^{\mathrm{aB}}$ & $0,88 \pm 0,18^{\mathrm{bC}}$ & $0,82 \pm 0,22^{\mathrm{bC}}$ \\
\hline Uva & $0,68+0,15^{\mathrm{bA}}$ & $0,67 \pm 0,20^{\mathrm{bA}}$ & $0,65 \pm 0,10^{\mathrm{cA}}$ & $0,61 \pm 0,12^{\mathrm{cA}}$ \\
\hline
\end{tabular}

*Médias \pm desvios padrões das análises realizadas em triplicata. a, b... (coluna): médias seguidas de mesma letra minúscula não diferem pelo teste de Tukey $(\mathrm{p}>0,05)$. A, B... (linha): médias seguidas de mesma letra maiúscula não diferem pelo teste de Tukey $(\mathrm{p}>0,05)$.

Tabela 3. Valores médios de sólidos solúveis totais ( $\left.{ }^{\circ} \mathrm{Brix}\right)$ em sucos de frutas de diferentes marcas durante o armazenamento sob refrigeração.

\begin{tabular}{|c|c|c|c|c|}
\hline \multirow{2}{*}{ Amostras } & \multicolumn{4}{|c|}{ Tempo de armazenamento sob refrigeração (dias) } \\
\hline & 0 & 7 & 14 & 21 \\
\hline \multicolumn{5}{|l|}{ Marca A } \\
\hline Caju & $13,00 \pm 0,10^{\mathrm{cB}}$ & $13,25 \pm 0,12^{\mathrm{cA}}$ & $13,25 \pm 0,12^{\mathrm{cA}}$ & $13,00 \pm 0,12^{\mathrm{cB}}$ \\
\hline Laranja & $13,00 \pm 0,16^{\mathrm{cB}}$ & $13,25 \pm 0,20^{\mathrm{cA}}$ & $12,75 \pm 0,10^{\mathrm{dC}}$ & $12,75 \pm 0,14^{\mathrm{aC}}$ \\
\hline Maracujá & $13,75 \pm 0,12^{\mathrm{bA}}$ & $13,50 \pm 0,16^{\mathrm{bB}}$ & $13,75 \pm 0,16^{\mathrm{bA}}$ & $13,75 \pm 0,20^{\mathrm{bA}}$ \\
\hline Uva & $14,25+0,08^{\mathrm{aB}}$ & $14,25 \pm 0,22^{\mathrm{aB}}$ & $14,75 \pm 0,10^{\mathrm{aA}}$ & $14,75 \pm 0,10^{\mathrm{aA}}$ \\
\hline \multicolumn{5}{|l|}{ Marca B } \\
\hline Caju & $11,25 \pm 0,10^{\mathrm{dD}}$ & $11,75 \pm 0,12^{\mathrm{dA}}$ & $11,50 \pm 0,12^{\mathrm{cC}}$ & $11,75 \pm 0,12^{\mathrm{dB}}$ \\
\hline Laranja & $12,25 \pm 0,16^{\mathrm{cA}}$ & $12,12 \pm 0,20^{\mathrm{cB}}$ & $12,25 \pm 0,10^{\mathrm{bA}}$ & $12,25 \pm 0,14^{\mathrm{bA}}$ \\
\hline Maracujá & $13,25 \pm 0,12^{\mathrm{bA}}$ & $12,25 \pm 0,16^{\mathrm{bC}}$ & $12,25 \pm 0,16^{\mathrm{bC}}$ & $12,00 \pm 0,20^{\mathrm{cB}}$ \\
\hline Uva & $14,25+0,08^{\mathrm{aA}}$ & $13,75 \pm 0,22^{\mathrm{aB}}$ & $13,00 \pm 0,10^{\mathrm{aC}}$ & $13,00 \pm 0,10^{\mathrm{aC}}$ \\
\hline \multicolumn{5}{|l|}{ Marca C } \\
\hline Caju & $13,25 \pm 0,10^{\mathrm{bA}}$ & $12,25 \pm 0,12^{\mathrm{cC}}$ & $12,25 \pm 0,12^{\mathrm{cC}}$ & $12,75 \pm 0,12^{\mathrm{bB}}$ \\
\hline Laranja & $12,75 \pm 0,16^{\mathrm{cA}}$ & $12,75 \pm 0,20^{\mathrm{bA}}$ & $12,50 \pm 0,10^{\mathrm{bC}}$ & $12,00 \pm 0,14^{\mathrm{cB}}$ \\
\hline Maracujá & $13,00 \pm 0,12^{\mathrm{bA}}$ & $12,25 \pm 0,16^{\mathrm{cB}}$ & $12,25 \pm 0,16^{\mathrm{cB}}$ & $12,00 \pm 0,20^{\mathrm{bC}}$ \\
\hline Uva & $14,15+0,08^{\mathrm{aA}}$ & $13,10 \pm 0,22^{\mathrm{aA}}$ & $13,00 \pm 0,10^{\mathrm{aB}}$ & $13,00 \pm 0,10^{\mathrm{cB}}$ \\
\hline
\end{tabular}

*Médias \pm desvios padrões das análises realizadas em triplicata. a, b... (coluna): médias seguidas de mesma letra minúscula não diferem pelo teste de Tukey $(\mathrm{p}>0,05)$. A, B... (linha): médias seguidas de mesma letra maiúscula não diferem pelo teste de Tukey $(\mathrm{p}>0,05)$.

Na abertura da embalagem, o suco de caju apresentou teores de SST variando de $11,25{ }^{\circ}$ Brix (marca B) a 13,25 ${ }^{\circ}$ Brix (marca C); o suco de laranja de $12,25^{\circ}$ Brix (marca B) a 13,00 ${ }^{\circ}$ Brix (marca A); o suco de maracujá de 13,00 ${ }^{\circ}$ Brix (marca C) a $13,75^{\circ}$ Brix (marca A); e o suco de uva de 14,15 ${ }^{\circ}$ Brix (marca C) a $14,25^{\circ}$ Brix (marcas A e B).

De acordo com os valores apresentados (Tabela 3), verificou-se que os sucos, desde o tempo inicial até os 21 dias durante o armazenamento, atenderam aos parâmetros mínimos de qualidade exigidos pela Instrução Normativa $n^{\circ}$ 01 de 7 de janeiro de 2000, do Ministério da Agricultura e do Abastecimento (BRASIL, 2000), que estabelece para os sucos industrializados os seguintes limites: SST, mínimo de 10,0 ${ }^{\circ}$ Brix para suco de caju; $10,5^{\circ}$ Brix para suco de laranja; 11,0 ${ }^{\circ}$ Brix para suco de maracujá e $14,00{ }^{\circ}$ Brix para suco de uva.

Nas Tabelas 4 e 5 observam-se o teor médio de ácido ascórbico (AA) nas amostras de sucos de frutas industrializadas e prontas para o consumo, logo após a abertura das embalagens, bem como o volume de suco necessário para atingir a ingestão dietética de referência (IDR) de $90 \mathrm{mg}$ para homens e $75 \mathrm{mg}$ para mulheres, a partir de 19 anos de idade (INSTITUTE OF MEDICINE, 2000). Observou-se que a variabilidade entre os teores de ácido ascórbico das amostras, que se apresentaram entre 20,34 $\mathrm{mg} / 100 \mathrm{~mL}$ para o suco de uva da marca C a $86,37 \mathrm{mg} / 100$ $\mathrm{mL}$ para o suco de caju da marca $\mathrm{B}$.

Os resultados do presente estudo revelam que para atingir o valor da IDR para homens e mulheres a partir de 19 anos, seria necessária a ingestão de um volume de suco variável entre 104 a $442 \mathrm{~mL}$ e de 87 a $369 \mathrm{~mL}$, respectivamente. Desta forma, mesmo para a amostra que apresentou o menor teor de AA, a ingestão diária de dois a três copos de suco $(200 \mathrm{~mL})$ seria suficiente para suprir a IDR no dia (INSTITUTE OF MEDICINE, 2000). 
Tabela 4. Teor médio inicial de ácido ascórbico $(\mathrm{mg} / 100 \mathrm{~mL})$ em sucos de frutas de diferentes marcas durante o armazenamento sob refrigeração e o volume de suco necessário para atingir a IDR.

\begin{tabular}{|c|c|c|c|}
\hline \multirow{2}{*}{ Sucos de frutas industrializados } & \multirow{2}{*}{$\begin{array}{c}\text { Teor de AA } \\
(\mathrm{mg} / 100 \mathrm{~mL})\end{array}$} & \multicolumn{2}{|c|}{ Volume de suco $(\mathrm{mL})^{*}$} \\
\hline & & Homens & Mulheres \\
\hline \multicolumn{4}{|l|}{ Marca A } \\
\hline Caju & $68,04 \pm 0,10^{\mathrm{b}}$ & 132 & 110 \\
\hline Laranja & $72,10 \pm 0,12^{\mathrm{a}}$ & 125 & 104 \\
\hline Maracujá & $48,40+0,21^{\mathrm{c}}$ & 186 & 155 \\
\hline Uva & $72,68+0,18^{\mathrm{a}}$ & 124 & 103 \\
\hline \multicolumn{4}{|l|}{ Marca B } \\
\hline Caju & $80,34 \pm 0,14^{\mathrm{b}}$ & 112 & 93 \\
\hline Laranja & $86,37 \pm 0,18^{\mathrm{a}}$ & 104 & 87 \\
\hline Maracujá & $60,06 \pm 0,20^{\mathrm{c}}$ & 150 & 125 \\
\hline Uva & $60,38 \pm 0,16^{\mathrm{c}}$ & 149 & 124 \\
\hline \multicolumn{4}{|l|}{ Marca C } \\
\hline Caju & $30,20 \pm 0,11^{\mathrm{b}}$ & 298 & 248 \\
\hline Laranja & $46,10 \pm 0,14^{\mathrm{a}}$ & 195 & 163 \\
\hline Maracujá & $25,50 \pm 0,22^{\mathrm{c}}$ & 353 & 294 \\
\hline Uva & $20,34 \pm 0,12^{\mathrm{d}}$ & 442 & 369 \\
\hline
\end{tabular}

Médias seguidas pela mesma letra na coluna não diferem pelo teste de Tukey $(\mathrm{p}>0,05)$. *Ingestão dietética de referência (IDR = $90 \mathrm{mg})$ para homens $\mathrm{e}$ (IDR $=75 \mathrm{mg}$ ) para mulheres a partir de 19 anos de idade.

Dados de pesquisas revelam que os sucos prontos para o consumo estão presentes em $30 \%$ dos lares brasileiros. Assim, sendo, em estudo realizado por Moreira et al. (2012), sobre os teores de AA em sucos e refrescos de tangerina, concluíram que os sucos estudados demonstraram a importância de seu valor nutritivo, principalmente no que diz respeito ao atendimento às recomendações nutricionais, uma vez que apresentam crescente aceitação pela população brasileira.

Os sucos processados têm vida útil extensa quando comparados ao suco fresco. Contudo, podem ocorrer perdas de nutrientes em função das condições de armazenamento. A importância nutricional dos sucos de fruta tem motivado a realização de estudos que visam estimar o comportamento da vitamina $\mathrm{C}$ durante a estocagem (TEIXEIRA; MONTEIRO, 2006). Nesse sentido, o ácido ascórbico tem sido usado como um importante marcador ou indicador da qualidade de sucos de fruta.
Verifica-se na Tabela 5 a variação no teor médio de AA dos diferentes tipos de sucos de frutas industrializados, em 21 dias de armazenamento sob refrigeração a $7^{\circ} \pm 2^{\circ} \mathrm{C}$. Verificase que desde o momento da abertura das embalagens os teores de ácido ascórbico diferiram significativamente entre os tratamentos. Observa-se, ainda, que os teores de ácido ascórbico nas amostras de sucos de frutas, diminuíram a partir do $7^{\circ}$ dia de armazenamento até o final, apresentando diferentes níveis na estabilidade do ácido ascórbico.

Para se avaliar a efetividade do ácido ascórbico, a mesma pode ser expressa em porcentagem de perda ou de retenção após o tempo e demais condições padronizadas. Portanto, para facilitar a análise, os mesmos resultados expressos na Tabela 5 foram apresentados em porcentagem de estabilidade do ácido ascórbico residual na Tabela 6 .

Tabela 5. Valores de ácido ascórbico $(\mathrm{mg} / 100 \mathrm{~mL})$ em sucos de frutas de diferentes marcas durante o armazenamento sob refrigeração.

\begin{tabular}{|c|c|c|c|c|}
\hline \multirow{2}{*}{ Amostras } & \multicolumn{4}{|c|}{ Tempo de armazenamento sob refrigeração (dias) } \\
\hline & 0 & 7 & 14 & 21 \\
\hline \multicolumn{5}{|l|}{ Marca A } \\
\hline Caju & $68,04 \pm 0,10^{\mathrm{bA}}$ & $60,10 \pm 0,08^{\mathrm{bB}}$ & $58,20 \pm 0,11^{\mathrm{bC}}$ & $40,10 \pm 0,10^{\mathrm{bD}}$ \\
\hline Laranja & $72,10 \pm 0,12^{\mathrm{aA}}$ & $68,34 \pm 0,10^{\mathrm{aB}}$ & $60,57 \pm 0,16^{\mathrm{aC}}$ & $52,12 \pm 0,14^{\mathrm{aD}}$ \\
\hline Maracujá & $48,40+0,21^{\mathrm{cA}}$ & $24,00 \pm 0,12^{\mathrm{cB}}$ & $16,40 \pm 0,20^{\mathrm{cC}}$ & $12,46 \pm 0,20^{\mathrm{dD}}$ \\
\hline Uva & $72,68+0,18^{\mathrm{aA}}$ & $60,70 \pm 0,16^{\mathrm{bB}}$ & $60,30 \pm 0,10^{\mathrm{aB}}$ & $20,26 \pm 0,12^{\mathrm{cC}}$ \\
\hline \multicolumn{5}{|l|}{ Marca B } \\
\hline Caju & $80,34 \pm 0,14^{\mathrm{bA}}$ & $69,25 \pm 0,15^{\mathrm{aB}}$ & $60,20 \pm 0,11^{\mathrm{aC}}$ & $58,10 \pm 0,10^{\mathrm{aD}}$ \\
\hline Laranja & $86,37 \pm 0,18^{\mathrm{aA}}$ & $75,59 \pm 0,22^{\mathrm{bB}}$ & $70,40 \pm 0,16^{\mathrm{bC}}$ & $70,18 \pm 0,08^{\mathrm{bC}}$ \\
\hline Maracujá & $60,06 \pm 0,20^{\mathrm{cA}}$ & $56,50 \pm 0,16^{\mathrm{cB}}$ & $54,38 \pm 0,15^{\mathrm{cC}}$ & $54,29 \pm 0,20^{\mathrm{cC}}$ \\
\hline Uva & $60,38 \pm 0,16^{\mathrm{cA}}$ & $53,25 \pm 0,10^{\mathrm{dB}}$ & $53,00 \pm 0,14^{\mathrm{dB}}$ & $50,10 \pm 0,16^{\mathrm{dC}}$ \\
\hline \multicolumn{5}{|l|}{ Marca C } \\
\hline Caju & $30,20 \pm 0,11^{\mathrm{bA}}$ & $30,00 \pm 0,15^{\mathrm{bB}}$ & $28,39 \pm 0,11^{\mathrm{bC}}$ & $20,50 \pm 0,10^{\mathrm{bD}}$ \\
\hline Laranja & $46,10 \pm 0,14^{\mathrm{aA}}$ & $46,02 \pm 0,22^{\mathrm{A}}$ & $40,00 \pm 0,16^{\mathrm{aB}}$ & $34,28 \pm 0,08^{\mathrm{aC}}$ \\
\hline Maracujá & $25,50 \pm 0,22^{\mathrm{cA}}$ & $23,30 \pm 0,16^{\mathrm{cB}}$ & $20,10 \pm 0,15^{\mathrm{cC}}$ & $18,00 \pm 0,20^{\mathrm{cD}}$ \\
\hline Uva & $20,34 \pm 0,12^{\mathrm{dA}}$ & $18,10 \pm 0,10^{\mathrm{dB}}$ & $18,05 \pm 0,14^{\mathrm{dB}}$ & $10,30 \pm 0,16^{\mathrm{dC}}$ \\
\hline
\end{tabular}

*Médias \pm desvios padrões das análises realizadas em triplicata. a, b... (coluna): médias seguidas de mesma letra minúscula não diferem pelo teste de Tukey ( $>$ > 0,05). A, B... (linha): médias seguidas de mesma letra maiúscula não diferem pelo teste de Tukey ( $>$ > 0,05). 
Tabela 6. Estabilidade do ácido ascórbico residual $(\%)$ em sucos de frutas de diferentes marcas durante o armazenamento sob refrigeração.

\begin{tabular}{|c|c|c|c|c|}
\hline \multirow{2}{*}{ Amostras } & \multicolumn{4}{|c|}{ Tempo de armazenamento sob refrigeração (dias) } \\
\hline & 0 & 7 & 14 & 21 \\
\hline \multicolumn{5}{|l|}{ Marca A } \\
\hline Caju & 100 & 88,33 & 85,54 & 58,94 \\
\hline Laranja & 100 & 94,78 & 84,00 & 72,29 \\
\hline Maracujá & 100 & 49,59 & 33,88 & 25,74 \\
\hline Uva & 100 & 83,52 & 82,97 & 27,88 \\
\hline \multicolumn{5}{|l|}{ Marca B } \\
\hline Caju & 100 & 86,20 & 74,93 & 72,32 \\
\hline Laranja & 100 & 87,52 & 81,51 & 81,25 \\
\hline Maracujá & 100 & 94,07 & 90,54 & 90,39 \\
\hline Uva & 100 & 88,19 & 87,78 & 82,97 \\
\hline \multicolumn{5}{|l|}{ Marca C } \\
\hline Caju & 100 & 99,34 & 94,00 & 67,88 \\
\hline Laranja & 100 & 99,83 & 86,77 & 74,36 \\
\hline Maracujá & 100 & 91,37 & 78,82 & 70,59 \\
\hline Uva & 100 & 88,99 & 88,74 & 50,64 \\
\hline
\end{tabular}

Em 7 dias de armazenamento, a degradação do ácido ascórbico apresenta-se de forma não significativa, ou seja, menor que $50 \%$. No $14^{\circ}$ dia os sucos estudados, ainda, obtiveram uma maior retenção da vitamina $\mathrm{C}$, com exceção do suco de maracujá (marca A) que se apresentou menor de $34 \%$. Observa-se ainda que, ao final do período de armazenamento, houve uma degradação do ácido ascórbico somente para os sucos de maracujá e uva (marca A). Para os demais sucos pode-se observar que apresentaram maior eficiência na retenção desta vitamina durante o período de estudo. Assim sendo, foi possível verificar que a maior estabilidade do ácido ascórbico para os sucos de frutas testados neste experimento in vitro foi para os sucos de laranja (marca A e C) e para o suco de maracujá (marca B).

Cunha et al. (2014) realizaram um estudo sobre a estabilidade de ácido ascórbico em sucos de frutas frescos sob diferentes formas de armazenamento. Concluíram que o ácido ascórbico foi estável por 24 horas no suco de laranja, porém apresentou decréscimo significativo após 8 horas de armazenamento nos sucos de abacaxi e melancia, possivelmente em virtude das diferenças de acidez inicial desses sucos. Em comparação com a literatura disponível, verificou-se que sucos frescos apresentaram menor estabilidade de ácido ascórbico do que sucos industrializados.

\section{CONCLUSÕES}

O pH, a acidez total e os sólidos solúveis totais, apresentam variações durante o armazenamento. A maioria das amostras analisadas de sucos atende aos parâmetros mínimos de qualidade exigidos pela Instrução Normativa do Ministério da Agricultura e do Abastecimento, que estabelece as diretrizes para a industrialização de polpas e sucos de frutas.

Os sucos de frutas comercializados apresentam retenção de ácido ascórbico após 21 dias de armazenamento sob refrigeração, indicando pequena redução de vitamina $\mathrm{C}$ durante o processamento.

\section{AGRADECIMENTOS}

À UEMG/CNPq/FAPEMIG pela concessão da bolsa de Iniciação Científica Júnior - BIC Jr.

\section{REFERÊNCIAS}

AOAC. Association of Official Analytical Chemists. Official and tentative methods of the Association of Official Analytical Chemists International. 18th ed. Maryland, 2005.

BRASIL. Ministério da Agricultura e do Abastecimento. Instrução Normativa $n^{\circ} 1$, de 7 de janeiro de 2000. Complementa padrões de identidade e qualidade para suco de laranja. Diário Oficial da União da República Federativa do Brasil. Brasília (DF), 10 jan 2000. Seção 1. p.54.

BRASIL. Ministério da Agricultura, Pecuária e Abastecimento. Decreto n 2.314, de 04 de setembro de 1997. Regulamenta a Lei ${ }^{\circ} 8.918$, de 14 de julho de 1994, que dispõe sobre a padronização, a classificação, o registro, a inspeção, a produção e a fiscalização de bebidas. Disponível em:<http://extranet.agricultura.gov.br/sislegisconsulta/consult arLegislacao.do?operacao=visualizar\&id=1010>. Acesso em agosto de 2015 .

CASTRO, M. V.; OLIVEIRA, J. P.; JÚNIOR, M. J. M.; ASSUNÇÃO, E. A. O.; BRASIL, A. P.; RABELO, F. L. A.; VALE, C. H. B. Análise química, físico-química e microbiológica de sucos de fruta industrializados. Diálogos \& Ciência, Rio de Janeiro, v. 5, n.12, 2007.

CECCHI, H. M. Fundamentos teóricos e práticos em análise de alimentos, $2^{\mathrm{a}}$ Ed. rev., Campinas, Editora da UNICAMP, 2003, 202p.

CORRÊA-NETO, R. S.; FARIA. J. A. F. Fatores que Influem na Qualidade do Suco de Laranja. Ciência e Tecnologia de Alimentos, Campinas, v.19, n.1, p.153-160, 1999.

CUNHA, K. D.; SILVA, P. R.; COSTA, A. L. F. S. F.; TEODORO, A. J.; KOBLITZ, M. G. B. Estabilidade de ácido ascórbico em sucos de frutas frescos sob diferentes formas de 
armazenamento. Brazilian Journal of Food Technology, Campinas, v.17, n.2, p.139-145, 2014.

DANIELI, F.; COSTA, L. R. L. G.; SILVA, L. C.; HARA, A. S. S.; SILVA, A. A. Determinação de vitamina $C$ em amostras de sucos in natura e amostras comerciais de suco de laranja pasteurizado e envasado em embalagem Tetra Pak. Revista do Instituto de Ciências da Saúde, São Paulo, v.27, n.4, p.361-365, 2009.

HOROWITZ, W. (Ed.). Official methods of analysis of the Association of Official Analytical Chemists. 18th ed., 3 rev. Gaithersburg, Maryland: AOAC, 2010.

INSTITUTE OF MEDICINE. Food and Nutrition Board Dietary Reference Intakes. National Academic Press, Washington D.C., 2000.

INSTITUTO ADOLFO LUTZ. Normas analíticas do Instituto Adolfo Lutz: métodos químicos e físicos para análises de alimentos. $4^{\text {a }}$ Ed. São Paulo, 2008. 1004p.

LIMA, E. S. Redução de vitamina C em suco de caju (Anacardium occidentale L.) industrializado e cajuína. Química Nova, São Paulo, v.30, n.5, p.1143-1146, 2007.

MAHAN, L. K.; ESCOTT-STUMP, S. Alimentos, nutrição \& dietoterapia. $11^{\text {a }}$ Ed. São Paulo: Roca; 2005. p. 35-71.

MOREIRA, C. F. F.; LOPES, M. L. M.; VALENTEMESQUITA, V. L. Impacto da estocagem sobre atividade antioxidante e teor de ácido ascórbico em sucos e refrescos de tangerina. Revista de Nutrição, Campinas, v.25, n.6, p.743$752,2012$.

ÖZKAN, M.; AYSEGÜL, K.; CEMEROGLU, B. Effects of hydrogen peroxide on the stability of ascorbic acid during storage in various fruit juices. Food Chemistry, Easton, v.88, p.591-597, 2004.

ROSA, S. E. S.; COSENZA, J. P.; LEÃO, L. T. S. Panorama do setor de bebidas no Brasil. BNDES Setorial, Rio de Janeiro, v.23, p.101-50, 2006.

SILVA, F. A. S. E.; AZEVEDO, C. A. V. Versão do programa computacional Assistat para o sistema operacional Windows. Revista Brasileira de Produtos Agroindustriais, Campina Grande, v. 4, n. 1, p. 71-78, 2002.

SILVA, P. T.; FIALHO, E.; LOPES, M. L. M.; VALENTEMESQUITA, V. L. Sucos de laranja industrializados e preparados sólidos para refrescos: estabilidade química e físico-química. Ciência e Tecnologia de Alimentos, Campinas, v. 25, n. 3, p. 597-602, 2005.

SPINOLA, V.; BERTA, B.; CÂMARA, J. S.; CASTILHO, P. C. Effect of Time and Temperature on Vitamin C Stability in Horticultural Extracts. UHPLC-PDA vs. iodometric titration as analytical methods. LWT - Food Science and Technology, London, v.50, n.2, p.489-495, 2013.

TEIXEIRA, M.; MOMTEIRO, M. Degradação da vitamina C em suco de fruta. Alimentos e Nutrição, Araraquara, v. 17, n. 2, p. 219-227, 2006. 